\title{
Les conséquences de la crise sanitaire sur les
} restaurants dans la ville de New-York : enquête sur une industrie en crise à travers l'exemple des serveuses racisées précaires

The Consequences of the Health Crisis on Restaurants in New York City: An Investigation of an Industry in Crisis With a Special Focus On Racialized and Precarious Waitresses

\section{Lucie Mezuret}

\section{OpenEdition}

\section{Journals}

Édition électronique

URL : https://journals.openedition.org/tourisme/4089

DOI : $10.4000 /$ tourisme.4089

ISSN : 2492-7503

Éditeur

Association Mondes du tourisme

Référence électronique

Lucie Mezuret, «Les conséquences de la crise sanitaire sur les restaurants dans la ville de New-York enquête sur une industrie en crise à travers l'exemple des serveuses racisées précaires ", Mondes du Tourisme [En ligne], 20 | 2021, mis en ligne le 15 décembre 2021, consulté le 17 décembre 2021. URL http://journals.openedition.org/tourisme/4089 ; DOI : https://doi.org/10.4000/tourisme.4089

Ce document a été généré automatiquement le 17 décembre 2021.

Mondes du tourisme est mis à disposition selon les termes de la licence Creative Commons Attribution - Pas d'Utilisation Commerciale - Pas de Modification 4.0 International. 


\title{
Les conséquences de la crise
} sanitaire sur les restaurants dans la ville de New-York : enquête sur une industrie en crise à travers l'exemple des serveuses racisées précaires

\author{
The Consequences of the Health Crisis on Restaurants in New York City: An \\ Investigation of an Industry in Crisis With a Special Focus On Racialized and \\ Precarious Waitresses
}

\section{Lucie Mezuret}

Depuis le début de la crise sanitaire, la chute des activités touristiques dans la ville de New York a entraîné une précarisation des métiers de la restauration, notamment celui de serveuse. La rareté du travail ainsi que l'absence d'allocations chômage ont accentué la précarité financière de ce métier dont le salaire est largement constitué de pourboires. Cette dépendance provoque une vulnérabilité et de nouvelles formes de pression et d'oppression de la part de la clientèle. En mars 2020, du fait des bouleversements dans le secteur touristique engendrés par la pandémie, je faisais l'hypothèse que la crise sanitaire allait entraîner une précarisation financière ponctuelle. Mais je supposais parallèlement qu'à l'instar de ce que l'on a pu constater en France chez les infirmières, une dynamique de revalorisation symbolique et pécuniaire allait s'enclencher chez tous les acteurs du monde de la restauration. Cela n'a cependant pas été le cas, la crise ayant été un amplificateur des inégalités déjà présentes. Il s'en est suivi une exacerbation des lacunes du système d'emploi des serveuses, et ce d'autant plus que ces dernières appartenaient à une population non 
blanche, ce qui était le cas de la plupart des interlocutrices concernées par l'enquête. Je me suis alors demandé pourquoi la crise avait joué ce rôle.

2 Cette note de recherche s'appliquera à montrer comment les serveuses new-yorkaises ont été impactées par la crise sanitaire actuelle, poussant nombre d'entre elles à quitter cette profession, et comment la crise a agi comme un révélateur des dominations et des inégalités de genre, de race et de classe, «[jetant la] lumière sur les inégalités qui ont toujours été là ", comme le résumait l'une des femmes rencontrées, Rachel, 33 ans, Noire américaine, célibataire vivant avec sa mère à Coney Island. La crise sanitaire a ainsi fonctionné comme une « fait social total » (Mauss, 1925), c'est-à-dire qu'elle a mis « en branle dans certains cas la totalité de la société et de ses institutions ».

3 La littérature sur la condition des serveuses aux États-Unis se limite à quelques ouvrages majeurs, deux publiés en 1991: Dishing It Out: Power and Resistance among Waitresses in a New-Jersey Restaurant de Greta Foff Paules et Dishing It Out: Waitresses and their Unions in the Twentieth Century de Dorothy Sue Cobble. Le premier examine les stratégies symboliques que les serveuses utilisent afin de contrer les différentes violences dont elles sont les victimes. Le second essai, plus historique, analyse le mouvement syndical des serveuses dans les années 1950. Un ouvrage plus récent, Nickel and Dimed: On (Not) Making It in America (Ehrenreich, 2001) étudie les «working poors" (travailleurs pauvres) et notamment leur possibilité de survie avec le salaire minimum. L'auteure a voyagé durant six mois dans des villes des États-Unis et y a cherché du travail en changeant d'identité. Une partie de son enquête l'a ainsi amenée à être serveuse et à découvrir les conditions de vie réelles de ses collègues.

4 La notion d'«intersectionnalité» sera mobilisée puisqu'elle est centrale pour comprendre les différentes strates de discriminations qui touchent les serveuses. Le terme d'intersectionnalité a été forgé en 1989 par l'universitaire afro-américaine Kimberlé Crenshaw qui, au départ, l'avait employé dans un but juridique. Elle a étudié au sein de plusieurs entreprises des situations de discrimination vis-à-vis des employée'es. L'exemple le plus représentatif de la notion d'intersectionnalité se trouve dans le cas, décrit par Crenshaw, de DeGraffenreid v. General Motors, procès datant de 1976. L'affaire débute lorsque six femmes noires attaquent en justice le constructeur automobile General Motors pour licenciement abusif. Avant 1964, date du Civil Rights Act, l'entreprise n'engageait pas de femmes noires. Lorsque General Motors décida de licencier du personnel du fait de la crise économique, les femmes noires furent les premières à perdre leur emploi car elles avaient été les dernières engagées. Lors du procès, elles font valoir cet argument et le fait qu'elles étaient noires et femmes. Mais elles n'obtiennent pas gain de cause car le juge estime qu'il faut que les plaignantes choisissent un seul type de discrimination (ici la race ou le genre). La cour conclut qu'il n'existe pas de discrimination liée à la race puisque General Motors emploie des hommes noirs. En ce qui concerne le genre, les juges se placent du côté de l'entreprise en affirmant que l'accusation ne peut être retenue puisque le constructeur automobile emploie des femmes blanches. De là, le terme d'intersectionnalité (de l'anglais intersectionality), devenue une notion sociologique, désigne la situation de personnes subissant simultanément plusieurs formes, plusieurs stratifications de domination ou de discrimination dans une société. Dans le cas qui nous intéresse ici, les employée's de restauration travaillant au service, dans la ville de New York, sont en grande majorité des femmes. Depuis mars 2020, les serveuses sont exposées à de nouveaux risques, tant 
physiques que psychologiques, qui touchent de façon disproportionnée les femmes racisées.

5 Cette note de recherche s'appuie sur l'enquête téléphonique que j'ai menée entre mars et mai 2020 auprès d'employée's de la restauration, et plus précisément de serveuses. Je devais partir aux États-Unis pour une enquête de terrain dans le cadre de ma thèse de sociologie sur la condition des serveuses mais, face à la fermeture des frontières, j'ai contacté la directrice de l'organisation One Fair Wage (OFW), Saru Jayaraman, pour lui proposer mon aide à distance afin de suivre la situation d'une manière ou d'une autre. OFW est une association non gouvernementale créée en 2014, qui a pour but de revendiquer un salaire minimum pour les employée's de la restauration. S. Jayaraman m'apprit alors l'existence d'une plateforme en ligne rassemblant des informations sur les employée's de la restauration afin de déterminer leur éligibilité à une aide de 213 dollars (somme symbolique car le salaire horaire est de 2,13 dollars de l'heure dans de nombreux États). L'association pouvait leur verser cette somme grâce à des levées de fonds. Elle les aidait également administrativement à obtenir les compensations financières de l'État. J'ai pu, au cours de ce volontariat, m'entretenir avec environ 60 serveuses, dont certains témoignages sont rapportés ici. Je suis également entrée en contact avec un professeur spécialiste de la restauration à l'Université de New York (NYU) Steinhardt, Krishnendu Ray. Cette note s'appuie aussi sur son témoignage, ainsi que sur les rapports les plus récents de OFW. Enfin, depuis juillet 2021, j'ai effectué sept entretiens via Zoom avec des serveuses membres de l'association non gouvernementale ROC United NY (Restaurant Opportunities Centers United). Cette association à but non lucratif cherche à protéger les employée's du secteur tertiaire. Je pouvais me permettre de leur poser des questions plus personnelles que dans le cadre du volontariat, et donc recueillir des réponses plus étayées. Toutes les personnes interrogées étaient des femmes noires américaines, en insécurité financière, de 30 ans et plus (la plus âgées ayant 50 ans), avec une famille à charge et vivant à New York.

\section{Un métier déjà précaire avant la crise sanitaire}

6 Tout d'abord, il faut souligner l'importance du secteur de la restauration dans une ville comme New York. On y compte environ 20000 restaurants, qui dépendent fortement du tourisme. La majeure partie des serveuses interrogées lors du volontariat m'ont parlé du plaisir qu'elles prenaient à exercer leur métier, qui leur permet notamment de rencontrer des gens d'autres pays et d'autres cultures: «J'adore rencontrer des touristes d'autres pays ", me confiait Maya; et les interactions avec certains clients manquaient à Sarah, les deux femmes se définissant comme des «des personnes sociables» («people persons»). Cependant, cette satisfaction s'agissant de certains aspects de leur travail n'empêche pas qu'on y enregistre de fortes inégalités sociales et économiques, selon le genre et la « race $»^{1}$. Ces disparités de salaires sont visibles à New York d'un quartier à l'autre. Pour le dire de façon schématique, les restaurants les plus prestigieux (où le salaire est plus important et les conditions de travail plus satisfaisantes) se situent dans les quartiers les plus fortunés (Manhattan). Les postes sont tenus par une majorité d'hommes blancs. Dans les restaurants qui se trouvent dans les quartiers les plus pauvres (Bronx, Queens), on trouve majoritairement des femmes racisées au service. Elles font parties des working poors, ou travailleurs pauvres, cités plus haut (Ehrenreich, 2001). On perçoit dès lors comment les différentes strates 
d'inégalité s'enchâssent et créent des discriminations salariales. Dans un récent rapport, ROC United estime que

$19 \%$ des travailleurs de la restauration vivent dans la pauvreté dans l'État de New York [...]. De ce fait, les employée'es de la restauration sont deux fois plus nombreux à dépendre des bons alimentaires ou de Medicaid [équivalent de la Sécurité sociale française] pour s'en sortir par rapport aux autres travailleurs de l'État. (ROC United, 2016)

7 Lors de mon enquête, de mars à mai 2021, j'ai pu constater que la plupart des serveuses étaient noires américaines et latinos. Je formule ici l'hypothèse que la plupart des serveuses ayant répondu à l'enquête ont les conditions de travail les plus précaires et que cela est corrélé au fait qu'il s'agisse de femmes racisées. Cette constatation s'est confirmée lors de ma seconde enquête, à l'image de Maya, d'origine hispanique, et de Sarah, noire américaine, vivant respectivement dans le Bronx et dans le Queens.

Par ailleurs, d'après le même rapport de ROC United, sur "plus de 200000 parents travaillant dans l'industrie de la restauration, 92000 d'entre eux sont des mères et presque la moitié $(45000)$ sont des mères célibataires ». Diandra, serveuse noire américaine de 33 ans, vivant dans le Bronx avec ses deux enfants témoigne: "c'est particulièrement dur d'être seule et d'avoir des enfants dans l'industrie de la restauration ». Elle explique que c'est un double problème du fait de l'absence de solutions de garde pour les enfants et de la réticence des employeurs à embaucher des femmes avec enfants car ils les considèrent, selon Diandra, peu fiables (" unreliable »). De fait, les femmes seules avec enfants sont doublement pénalisées, parce qu'il est difficile de faire garder les enfants sans argent et, inversement, de gagner sa vie sans faire garder ses enfants.

D'autre part, il existe un très grand contraste entre l'argent généré par la restauration aux États-Unis (chiffre d'affaires direct estimé à plus de 12 milliards de dollars annuels) et le salaire des serveuses. En effet, ces dernières touchent le "subminimum wage » (en dessous du salaire minimum) également appelé "tipped minimum wage», en référence au tip, c'est-à-dire le pourboire. La place dominante du système du pourboire dans le salaire des serveureuse's est une caractéristique propre aux États-Unis. Le salaire "subminimum » varie d'un État à l'autre, le minimum fédéral étant de 2,13 dollars (ce qui constitue le subminimum wage). Ce système est un héritage de l'esclavage et une coutume héritée de l'aristocratie européenne au XIX ${ }^{e}$ siècle. Cette pratique a ses racines dans l'Amérique de la fin de la guerre civile, après l'abolition de l'esclavage en 1865. Les Africains-Américains peuvent alors prétendre à un salaire. Cependant, peu de métiers leur sont accessibles. Ils se retrouvent souvent dans des emplois subalternes, tels que serveurs. Beaucoup d'employeurs leur refusent un salaire, déléguant de fait la rémunération aux clients. Malgré les plaintes d'États jugeant ce système injuste, celuici s'est répandu, notamment dans les États du Sud. Les employeurs comprirent rapidement les bénéfices qu'ils pouvaient tirer de cette pratique, qui se propagea ensuite dans les États du Nord (Greenspan, 2019). En 1938, le Fair Labor Standards Act (FLSA) est signé par Franklin D. Roosevelt pendant le New Deal et permet aux employeurs de payer seulement un faible salaire à leurs employé.e.s gagnant des "gratuities », c'est-à-dire des pourboires. Si la somme ne permet pas à l'employé.e de toucher le salaire minimum fédéral (7,25 dollars par heure, full minimum wage), l'employeur se doit de payer la différence, ce qu'il ne fait presque jamais. Comme le remarque Barbara Ehrenreich, cette partie de la loi ne lui a jamais été communiquée, ni même mentionnée dans les restaurants où elle a travaillé comme serveuse au cours de 
l'enquête qu'elle a menée en Floride (Ehrenreich, 2001). C'est ce qui s'appelle le « wage theft ", littéralement "vol de salaire ». Celui-ci est très rarement puni, notamment parce que les employée's ne connaissent pas la loi dans son entièreté. De plus, depuis 1966, les amendements au FLSA étendent et normalisent le système du pourboire (Allegretto et Cooper, 2014). Les conditions d'accès au salaire minimum n'ont pas changé depuis les années 1970. De fait, encore de nos jours, dans beaucoup d'États (Alabama, Géorgie, Indiana), ce sont les pourboires des clients qui constituent l'essentiel du salaire des serveur-euse's. Au cours du volontariat que j'ai effectué entre mars et mai 2020, une serveuse m'a confié qu'une fois les impôts prélevés sur son salaire, il ne lui restait que 0,75 dollars et que son salaire était de 40 dollars toutes les deux semaines (sans les pourboires).

Dans cette situation où il leur est impossible de faire des économies, les serveuses sont donc extrêmement dépendantes des pourboires pour leur survie quotidienne, ce qui rend difficile pour elles de changer d'employeur si les conditions de travail sont mauvaises.

11 La directrice de l'association non gouvernementale One Fair Wage, Saru Jayaraman, utilise une expression qui décrit parfaitement le sentiment d'insécurité qui découle du tipped minimum wage : « to live from tip to mouth », littéralement « vivre du pourboire à la bouche ». L'expression met en lumière l'incertitude de pouvoir se nourrir soi et sa famille au quotidien. Elle joue sur une autre expression américaine "from hand to mouth » qui signifie « avoir du mal à joindre les deux bouts ».

Un rapport de ROC United de 2020 constate que les femmes vivant dans les États où prévaut le subminimum wage sont $5 \%$ plus pauvres que les hommes, un chiffre qui tombe à $2 \%$ dans les États où le full minimum wage s'applique. Les personnes racisées sont en outre $1 \%$ plus pauvres que les personnes blanches avec le full minimum wage et $4 \%$ plus pauvres avec le subminimum wage. On peut conclure de ces chiffres que les femmes de couleur sont impactées plus fortement puisqu'elles sont à l'intersection de deux types de discrimination (racisme et sexisme).

13 Catherine Barnett, ancienne directrice de ROC United à New York, affirme que des études ont montré qu'au-delà des différences de salaires selon les quartiers, le montant des pourboires donnés à une serveureuse ne dépendait pas de la qualité du service mais de facteurs extérieurs, comme le genre ou la couleur de peau. Les hommes blancs perçoivent des pourboires plus importants que les femmes blanches, les serveuses racisées touchant les pourboires les plus faibles. Au niveau national, il y a un écart salarial de 4,79 dollars par heure entre une femme noire et un homme blanc (ROC United, 2020).

On voit bien, à travers l'exemple du pourboire, comment les différentes discriminations (de sexe et de "race ») se superposent et s'entrecroisent, impactant particulièrement les serveuses racisées. Rachel résume cette idée : «Pour être aussi directe que possible : c'était un environnement raciste et sexiste voilà ce que c'était !». Le salaire minimum pour les employée's touchant des pourboires dans l'État de New York, pourtant d'environ 10 dollars, n'est pas suffisant pour permettre aux serveuses de vivre dignement. Sarah m'a confié qu'elle compte en majorité sur les pourboires pour s'assurer un salaire décent et Maya cumule deux emplois (serveuse le jour et chauffeuse de taxi la nuit). Cette précarité est d'autant plus marquée dans une ville comme New York, où le coût de la vie est prohibitif. À titre d'exemple, les loyers pour un appartement d'une pièce sont en moyenne de 1200 dollars par mois. 
15 Avant la crise sanitaire, le tourisme était une source importante de revenus pour les serveur-euse's. Peu habitués au système des pourboires, les visiteurs ont tendance à être plus généreux que les New-yorkais. Pour les touristes désireux d'une expérience « authentique » des États-Unis, le diner est un passage obligé. Ces restaurants familiaux sont un microcosme de la société fantasmée des années 1950-1960 et sont ancrés dans l'imaginaire de l'American Dream. Le diner est un type de bâtiment-restaurant au départ préfabriqué, typique de l'Amérique du Nord, en particulier du Nord-Est des États-Unis. Il propose un grand choix de mets pour la plupart caractéristiques (hamburger, bacon, œuf, café), une atmosphère sobre, un comptoir et des horaires d'ouverture étendus, souvent 24 heures sur 24 . Les personnes employées dans ces établissements sont, pour la plupart, racisées et précaires.

Les phénomènes de précarité décrits plus haut ont été accentués par la chute du tourisme à New York. À partir de la mi-mars 2020, les frontières ont été fermées. De fait, à partir de cette date, la majorité des restaurants ont réduit ou cessé leur activité. En avril 2020, la NRA (National Restaurant Association, l'organisation la plus importante liée à la restauration aux États-Unis) a publié un rapport qui affirmait qu'au niveau national, huit millions d'employée's avaient été licenciés, soit deux employée's sur trois, et que quatre restaurants sur dix avaient fermé leurs portes.

\section{Précarisation et vulnérabilité exacerbées par la crise sanitaire}

Dans ce second temps de réflexion, nous allons nous pencher sur les conséquences de la crise du tourisme sur le monde la restauration, pour comprendre dans quelle mesure celle-ci impacte plus fortement les serveuses racisées à New York. Krishnendu Ray m'a affirmé que les quartiers les plus touchés économiquement par la crise actuelle étaient les plus pauvres (Bronx, Queens, qui rassemblent des populations largement racisées) car ils dépendaient fortement du secteur de la restauration. En 2020, 46,1\% des employée's du secteur vivent deux fois en dessous du seuil de pauvreté et $20 \%$ dépendent des bons alimentaires. À New York, $79 \%$ des salariée's touchant des pourboires sont de couleur et presque $58 \%$ sont des femmes (ROC United, 2020). L'approche de l'intersectionnalité nous permet de constater que les femmes racisées sont les plus impactées par la crise sanitaire puisqu'elles subissent la «double peine » d'être noire et femme.

Cette crise a également mis en lumière l'absence de sécurité de l'emploi, insécurité qui se double d'une pratique très fréquente aux États-Unis, le «at-will employment » que l'on pourrait traduire en français par «emploi de gré à gré». En France, les contrats d'embauche sont formalisés par écrit, signés par l'employeur et l'employée.e. Ce n'est pas le cas aux États-Unis où un accord verbal fait office de contrat de travail pouvant être rompu sans aucun préavis (ni de la part de l'employéee, ni de la part de l'employeur). Ainsi, une employéee peut donc être licenciéee du jour au lendemain sans aucune compensation financière. Cette "flexibilité » est pourtant l'un des aspects qui plait aux serveur-euse-s. Mes interlocutrices étaient, pour la plupart, initialement ravies de la possibilité de pouvoir changer de travail quand bon leur semblait. L'un des avantages de ce métier, d'après elles, est la facilité à trouver un emploi rapidement et 
d'en quitter un aussi rapidement. Cette flexibilité donne aux serveuses une certaine liberté de choisir, principe cardinal de l'imaginaire américain :

Quand j'aimais pas mon patron ou mes collègues, je partais juste. Je cherchais pendant deux, trois jours et je retrouvais. J'avais pas l'impression d'être obligée de rester. J'me sentais libre, tu vois? (Maya)

Elles savent cependant qu'elles ont peu de chance d'évoluer vers des postes plus avantageux financièrement et qu'elles peuvent se faire licencier plus facilement sans contrat de travail, à l'image de ce qui est arrivé à Sarah en mars 2020 :

Un jour, je suis juste allée travailler comme d'hab et quand je suis arrivée le restaurant était fermé. Du coup, j'ai appelé mon patron et il m'a dit qu'à cause de la crise, le resto était fermé jusqu'à nouvel ordre et qu'il me rappellerait... C'était y'a deux mois..

Selon le Bureau of Labour Statistics (BLS, l'équivalent de l'Insee), l'année 2020 a enregistré un record de chômage chez les femmes : 16,2 \% en avril 2020 selon le Wall Street Journal. Si bien que la crise du Covid a été appelée la "she-cession» (mot créé à partir de «she» voulant dire « elle » et de « recession»). De plus, les serveuses n'ont pu accéder, pendant des mois, aux allocations chômage. D'après un rapport de OFW, 60 \% des travailleurs touchant des pourboires ont affirmé ne pas avoir eu accès à l'assurance chômage car leur salaire était trop bas pour cela (OFW, 2021). Là encore, on constate la superposition de différentes discriminations affectant les serveuses.

21 Par ailleurs, les restaurants qui sont restés ouverts enregistrent un fort taux de contamination à la Covid-19. Un rapport des Centers for Disease Control and Prevention (un équivalent des agences régionales de santé françaises) affirme que les personnes ayant mangé dans un restaurant ont deux fois plus de chance de contracter le virus, ce qui affecte la fréquentation des établissements. Les serveureuse-s sont pourtant des travailleur'euse's essentielle's, qui contribuent à nourrir la population, comme Rachel le résume avec humour :

Les restaurants, ou tout ce qui touche à la nourriture, c'est essentiel, ça fait partie d'un truc essentiel. C'est pour ça que les restos vont pas s'envoler, parce que les gens voudront toujours manger mais ne voudront pas toujours cuisiner.

Les employée's de la restauration prennent soin d'une grande partie de la population et pourtant, selon un rapport de OFW, leurs employeurs ne leur ont pas systématiquement fourni de protections (masques, gants) (OFW, 2021). Selon ce même rapport, plus de $84 \%$ des employée's interrogée's affirment se trouver, à chaque service, à moins d'un mètre de distance d'un client qui ne porte pas de masque. En effet, seuls $28 \%$ des employée's ont expliqué que les consignes de sécurité dans leurs restaurants avaient été respectées. Les femmes et les travailleurs de couleur ont été impactés de manière disproportionnée. Face à la pénurie d'emplois et à la baisse des pourboires ( $83 \%$ des employée's affirment avoir subi une baisse significative de leurs pourboires), les serveuses rencontrées ont dû se résoudre à accepter des situations dangereuses. Diandra raconte ainsi : « Il [le restaurant] n'était pas aussi plein, donc essayer d'avoir la même somme de pourboires était plus compliqué ». De fait, les serveuses se trouvent dans une nécessité accrue de revenus et puisent dans leurs économies pour survivre. Or, ces économies servaient aussi à prendre soin de leurs proches (parents ou enfants). Lorsque j'ai discuté avec Maya, elle était à l'hôpital pour rendre visite à sa mère et me confiait qu'elle ne savait pas comment régler la facture. Ces femmes ont dû prendre le risque de tomber malade et/ou de mettre en danger leurs proches : 
J'ai limite peur de rentrer chez moi maintenant. Je passe trente minutes à me laver, à me désinfecter, à me changer tous les soirs et j'ai quand même la trouille. J'ai pas envie de mettre en danger toute ma famille, mais d'un autre côté faut bien qu'on mange et qu'on paie les factures ! C'est pas les petits qui vont s'en charger ! (Sarah)

Autre conséquence : devant l'impossibilité de refuser les postes encore vacants, malgré les risques sanitaires, une " lutte des places » a vu le jour et entamé la solidarité entre serveuses, qui était pourtant une pierre angulaire de la défense de ces femmes face aux comportements déplacés qui n'ont fait que s'accroître depuis mars 2020. En effet, depuis le début de la pandémie, on constate une forte augmentation de la violence et de l'agressivité de la part des clients envers les employée's de la restauration. Diandra décrit la période de la Covid 19 comme éprouvante pour les nerfs ( " nerve wracking»). non-port du masque. L'association OFW a publié un rapport alarmant sur ces nouveaux comportements (MaskualHarassment Report). L'industrie de la restauration était déjà l'un des secteurs les plus touchés par le harcèlement sexuel. Selon un sondage de ROC United, 80 \% des employée's affirment avoir eu affaire à des commentaires salaces, des attouchements, etc. de la part des clients (ROC United, 2014). Cependant, depuis le début de la crise, $51 \%$ des mères de famille ont constaté une forte augmentation des comportements violents liés au port ou au non-port du masque. Sarah comme Maya m'ont affirmé qu'elles recevaient quotidiennement des insultes et des menaces de mort, menaces par exemple proférées lorsque la serveuse demande au client de remettre son masque correctement. De plus, $59 \%$ des mères affirment être réticentes à demander aux clients de porter un masque de peur qu'ils ne leur donnent pas de pourboires généreux. S'ajoute donc à cette violence le harcèlement sexuel lié au pourboire. Plus de $40 \%$ des femmes interrogées ont remarqué une intensification de la fréquence des « commentaires à caractère sexuel non désirés de la part des clients ». La relation de pouvoir entre le client et la serveuse est exacerbée par la crise sanitaire. Cette relation dominant/dominé est en grande partie liée à des questions de genre et de classe. Les serveuses se trouvant déjà dans une position de dominées le sont d'autant plus avec la Covid-19 et leur besoin d'un salaire décent, donc de pourboires. Dans son rapport, l'association OFW a compilé différentes phrases que les serveuses ont à entendre: les commentaires vont de "enlève ton masque que je sache combien te donner de pourboire » à " enlève ton masque que j'enfonce ma langue dans ta gorge » (OFW, 2021). C'est ce que l'association OFW a nommé le "Maskual Harassment " (expression composée de «mask» et de «sexual» pour faire référence au sexual harassment). Conscients de la vulnérabilité accrue de ces femmes, les clients transgressent les codes de bienséance habituellement respectés.

La crise sanitaire a entraîné la fermeture des frontières. L'une des conséquences de la chute du tourisme a été l'accroissement de la précarité des employée's de la restauration. Les serveuses se trouvent alors avec moins de ressources pour se défendre contre un harcèlement sexuel lui aussi en augmentation. Depuis mars 2020, beaucoup de femmes (notamment les mères de famille racisées) envisagent de quitter le métier : $80 \%$ des mères de famille travaillant dans la restauration affirment que la raison première en est le faible salaire et le système du pourboire ; $58 \%$ affirment que leur départ serait aussi motivé par l'insécurité face à la Covid-19. Faute d'un salaire décent, de protections sociales, d'une reconnaissance de la valeur de leur métier et de leur statut de travailleuse, les serveuses considèrent que l'industrie "n'en vaut plus la peine » (« it's just not worth it anymore »). De plus, depuis mi-septembre le pass sanitaire 
est obligatoire dans les restaurants et nombre de serveuses noires américaines refusent, pour des raisons historiques et par défiance à l'égard de l'État fédéral, de se faire vacciner. Ces femmes préfèrent risquer de perdre leur emploi. On voit ici comment la crise a fonctionné comme un amplificateur des représentations et des réalités des populations précarisées.

\section{BIBLIOGRAPHIE}

\section{Rapports}

OFFICE OF THE NEW YORK STATE COMPTROLLER, Budgetary/Statutory Basis Annual Report, 30 juin 2020. ONE FAIR WAGE, Take Off Your Mask So I Know How Much to Tip You, janvier 2021. ONE FAIR WAGE, Decline in Open Hospitality Businesses Due to COVID-19 A State-By-State Analysis, février 2021.

ONE FAIR WAGE, Ending A Legacy of Slavery. How Biden's COVID Relief Plan Cures the Racist Subminimum Wage, février 2021.

ONE FAIR WAGE, It's a Wage Shortage, not a Worker Shortage: Why Restaurant Workers, Especially Mothers, are Leaving the Industry and What Would Make Them Stay, mai 2021.

NRA, The Restaurant Industry. Impact Survey, National Restaurants Association, avril 2020.

NEW YORK CITY COUNCIL, « Council Votes to Prevent Fast Food Workers From being Fired Without "Just Cause" ", 17 décembre 2020.

ROC UNITED, State Of the Restaurant Workers Report, 2020 [https://stateofrestaurantworkers.com/].

ROC UNITED, Nightcare: The Growing Challenge for Parents on the Late Shift, 2016 [https://

chapters.rocunited.org/publications/nightcare-the-growing-challenge-for-parents-on-the-lateshift/].

ROC UNITED, The Glass Floor: Sexual Harassment in the Restaurant Industry, 2014 [https://

chapters.rocunited.org/publications/the-glass-floor-sexual-harassment-in-the-restaurantindustry/].

\section{Essai}

Barbara EHRENREICH, Nickel and Dimed: On (not) Getting by in America, Picador, 2011.

\section{Entretiens}

Maya : 35 ans, origine hispanique, vit dans le Bronx, mère à charge.

Sarah : 27 ans, noire américaine, vit dans le Queens, mère célibataire (deux enfants en bas âge).

Diandra : 33 ans, noire américaine, mère célibataire (deux enfants de 17 et 12 ans), travaille et vie dans le Bronx. 
Rachel : 33 ans, noire américaine, célibataire (sans enfant), vie avec sa mère (afro-caribéenne) et son père (afro-américain); son frère travaillait dans l'industrie de la restauration ; travaille à Coney Island, vit à Coney Island (première raison pour laquelle elle a pris le travail).

Ray Krishnendu : professeur à l'Université de New York (NYU), mars 2020.

\section{NOTES}

1. Aux États-Unis, le terme de "race » est couramment utilisé, à la fois pour s'autodésigner et dans les recensements officiels de la population qui offrent la possibilité de choisir entre plusieurs catégories d'appartenance : « White, Black or African American, American Indian, Asian, two or more races, Native Hawaiian ».

\section{AUTEUR}

\section{LUCIE MEZURET}

Sociologie

Doctorante

Université de Paris, LCSP

lucie.mezuret[at]hotmail.fr 